\title{
Recurrent Large Pericardial Effusion and Tamponade in a Young Patient with Erdheim Chester Syndrome: A Case Report and Review of the Syndrome
}

\author{
Walid Hassan*, Leena Waqar Ahmed, Tarek Tabbaa and Mariam Hassan \\ Department of Cardiovascular Disease, International Medical center, Saudi Arabia \\ Submission: August 18, 2017; Published: August 28, 2017
}

*Corresponding author: Walid Hassan, Department of Cardiovascular Disease, International Medical center, Saudi Arabia, Tel: + 966-126-509-000

; Fax: + 966-126-509-789; Email: whassan@imc.med.sa

\begin{abstract}
In this report, we present a case of young male with Erdheim Chester Syndrome, who presented with dyspnea due to recurrent large pericardial effusion and impending tamponade. The patient underwent percutaneous pericardiocentesis and started on steroid, interferon alpha and immunosuppressive treatment with subjective improvement of his symptoms and rate of pericardial fluid re accumulation. This report will give an updated review of the syndrome.
\end{abstract}

\section{Introduction}

Erdheim Chester Disease (ECD) is a Non- Langerhans histiocytic disorder without a clear cut etiology [1,2]. NonLangerhans histiocytic disorders are named as such due to the fact that the disorder or the cells affected are derived from cells other than that of Langerhans- typically of monocytic/ macrophagic origin [3]. Erdheim Chester Disease causes inflammation followed by invasion and accumulation in the tissue or organ involved and has been recently classified by the World Health Organization (WHO) as a histiocytic neoplasm [1-7]. It most commonly affects bones in the lower limbs; nevertheless, signs and symptoms may arise in the brain, lungs, heart, eyes, and other body organs [7]. Herein we present a case of 43 year-old male with ECS who presented with impending pericardial tamponade and managed with pericardiocentesis, steroids and immunosuppressant therapy.

\section{Case Presentation}

43 years old male with history of smoking, Erdheim Chester syndrome, hypertension, chronic renal impairment, recurrent urinary tract infection, bilateral urinary stenting, with several hospital admission over the last 5 years for bone aches, dyspnea, pleural and pericardial effusion required treatment with steroid, pleurocentesis and pericardiocentesis. In this current index admission he developed severe dyspnea and again was found to have large pericardial effusion with signs of tamponade.

\section{Vital signs}

Blood pressure 92/44mm Hg, pulse rate 118 beat/minute, Height $170 \mathrm{~cm}$, Weight $69 \mathrm{~kg}$. He has bilateral exophthalmoses and xanthelasma.

\section{Cardio vascular System}

Distant S1S2 no added sound and no mummer, Chest: fair air entry bilaterally with no added sound, Abdomen: soft, lax, lower extremities: tendon xanthiums. Laboratory investigations showed abnormal complete blood count \& renal function white blood cells (WBCs) 12.4, hemoglobin $10.9 \mathrm{gm} / \mathrm{dl}$, hematocrite $33 \%$, uric acid $11 \mathrm{mg} / \mathrm{dl}$, serum creatinine $2.2 \mathrm{mg} / \mathrm{dl}$, glomular filteration rate (GFR) 32. Initial High Sensitivity C-Reactive protein (hs-CRP) was elevated $352 \mathrm{mg} / \mathrm{l}$ (normal<5) and in follow up normalized $7.0 \mathrm{mg} / \mathrm{l}$. Electrocardiography showed sinus tachycardia, heart rate 118 beat/minute and nonspecific anterior ST-T changes.

Chest $\mathrm{X}$ ray on presentation revealed cardiomegaly (flask-shape heart) (Figure 1a \& 1b) Echocardiography on presentation; Revealed normal left ventricular systolic function, ejection fraction 55-60\%, no significant valve disease, and very large pericardial effusion with tamponade signs including diastolic right atrial and right ventricular collapse (Figure 2a). He underwent percutaneous pericardiocentesis with removal 
of $1400 \mathrm{cc}$ of serious fluid that was proven to be inflammatory transudate by fluid analysis with several macrophages and histiocytes in the fluid. Post pericardiocentesis echocardiography showed minimal effusion and no tamponade physiology (Figure $2 b)$. He was maintained on oral corticosteroid, interferon alpha and immunosuppressant therapy, follow up clinic visits after 3 , 6 and 9 months revealed improvement of his symptoms and no further effusion or re accumulation by repeat chest $\mathrm{X}$ ray and echocardiography after 9 months.

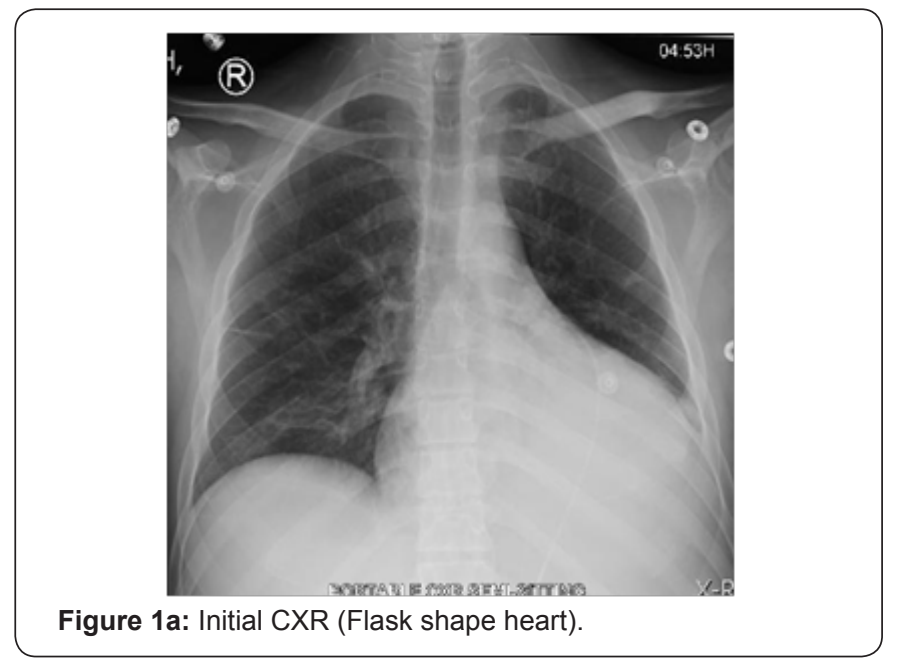

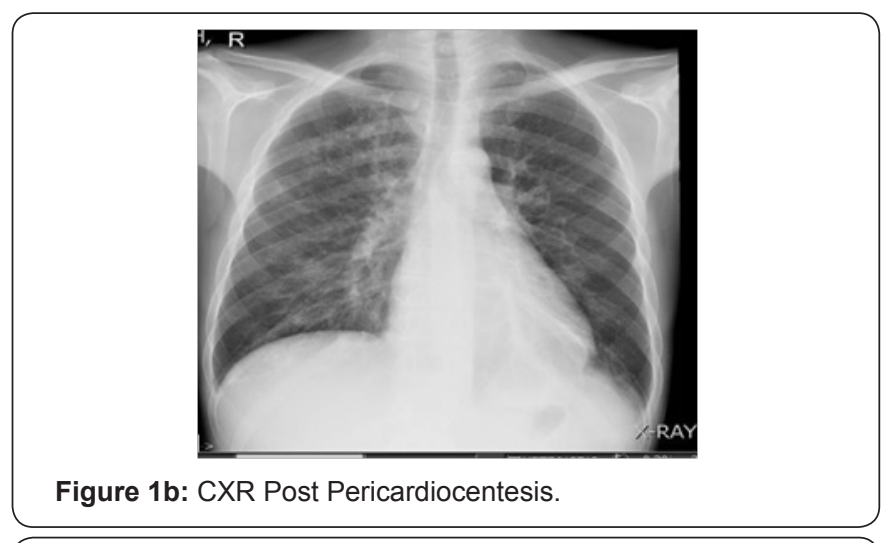

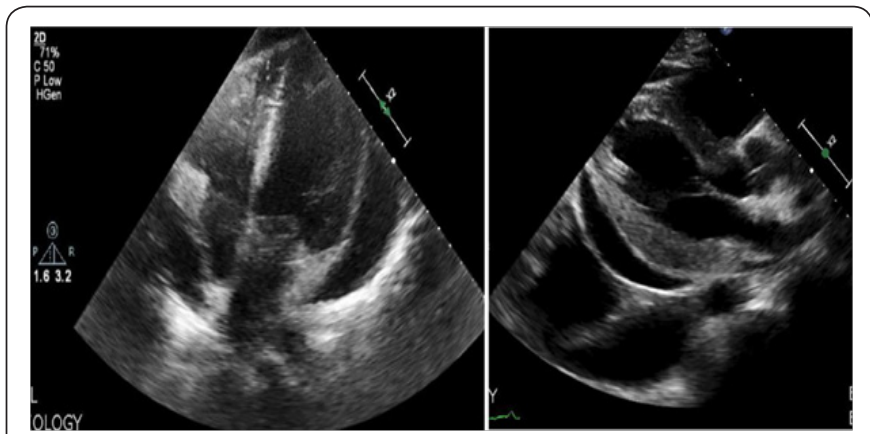

Figure 2a: Initial echocardiography revealing large effusion, diastolic RV and RA collapse.

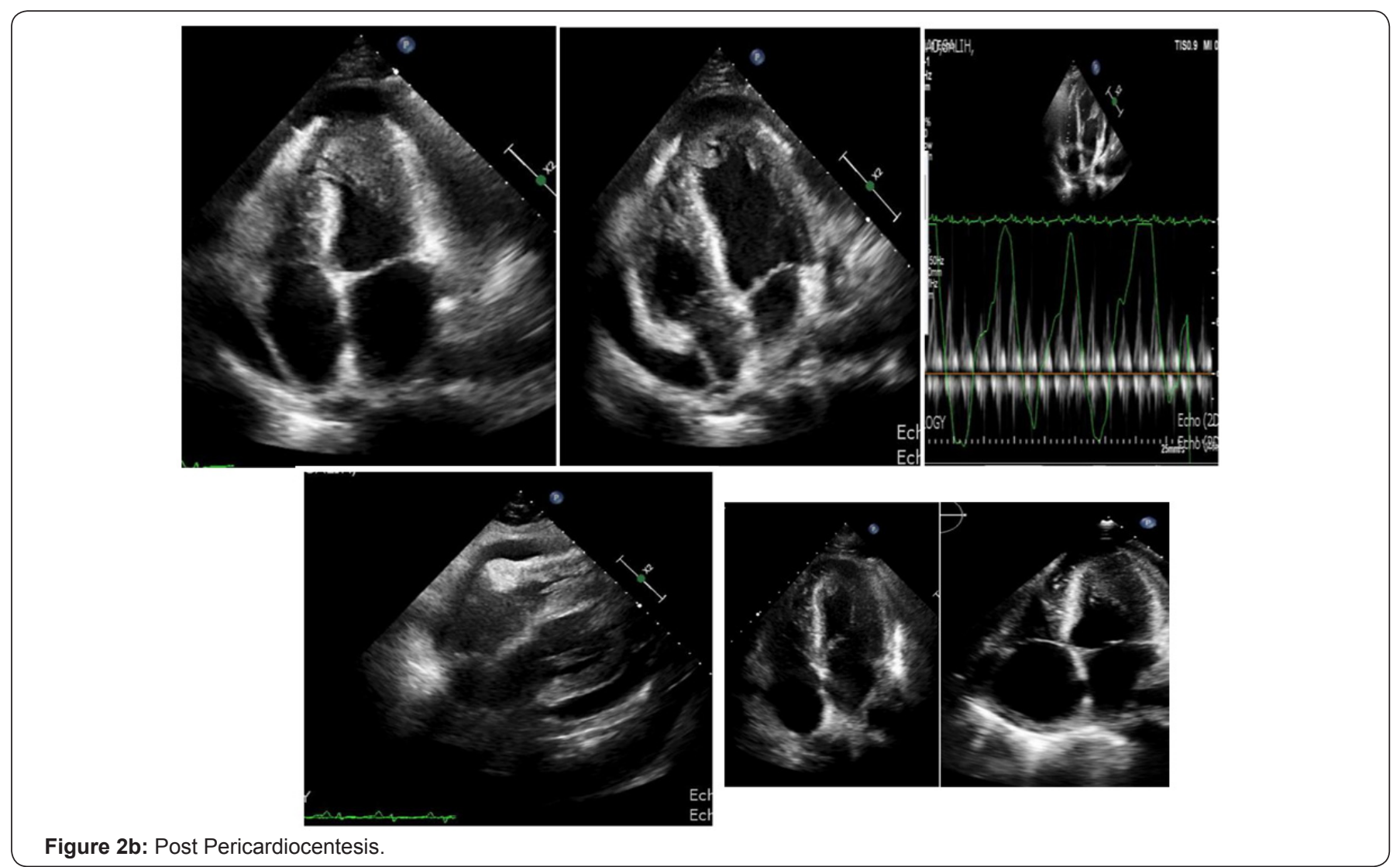

\section{Discussion}

Histiocytes are a type of leukocytes. Histiocytic disorder is a condition that is characterized by excessive proliferation of histiocytes. Erdheim Chester Disease (ECD) is a Non-
Langerhans histiocytic disorder without a clear cut etiology $[1,2]$. Non- Langerhans histiocytic disorders are named as such due to the fact that the disorder or the cells affected are derived from cells other than that of Langerhans- typically of monocytic/ 
macrophagic origin [3]. Langerhans cells are dendritic cells (antigen-presenting cells) found abundantly in the epidermis [3-5]. ECD causes inflammation followed by invasion and accumulation in the tissue or organ involved and has been recently classified by the World Health Organization (WHO) as a histiocytic neoplasm $[1,6]$. This results in thickening, fibrosis, and eventual necrosis or failure.

It most commonly affects bones in the lower limbs; nevertheless, signs and symptoms may arise in the brain, lungs, eyes, and other body organs [7]. According to the Erdheim Chester Global Alliance, ECD is usually diagnosed between the fourth and seventh decade in one's lifespan and for unknown reasons; there is a higher rate of incidence in males. ECD is considered a rare disease with less than a few hundred cases reported, yet it is considered under diagnosed due the complexity of its etiology and pathophysiology and difficulty of its identification and recognition and treatment $[1,3,4,8,9]$.

\section{Causes}

As aforementioned, the causes of occurrence of ECD are unclear and can alter from one patient to another. Nonetheless, several key features have been identified in most patients diagnosed. For instance, BRAF gene (a proto oncogene), has been identified to be somatically mutated in $38-68 \%$ of patients as reported in a certain research. $[10,11]$. BRAF gene encodes for serine/threonine kinase B-RAF protein which regulates the ERK (aka Mitogen-Activated Protein Kinase) pathway and is responsible for transmitting chemical signals from the extracellular environment to the intranuclear area [12,13]. The ERK pathway is responsible for cell division and other important procedures [13]. The mutation in BRAF that might occur in ECD patients results in the replacement of a single amino acid in the primary sequence. It replaces valine by glutamic acid in position 600 (Val600Glu).

The mutation leads the conversion of the proto-oncogene to an oncogene and hence an overactive protein (B-RAF protein). The over activity of the protein disrupts regulation of cell proliferation and hypertrophy allowing histiocytes to grow and divide uncontrollably [7]. On another note, histiocytes in ECD cases tend to promote inflammatory responses through the usage of chemokines and cytokines resulting in the application and activation of more histiocytes in the inflamed area. Furthermore, ECD results in increasing the level of interferon alpha (IFN- $\alpha$ ), monocyte chemotactic protein-1 (MCP-1), and interleukin- 12 as well as decreasing the levels of interleukins 4 and 7 [10].

\section{Symptoms}

Erdheim-Chester disease usually manifests as bone pain in about $26 \%$ of cases [13]. However, patients can also present with non-osseous involvement depending on the affected site. Thus, apart from bone pain, patients might also present with neurological features, diabetes insipidus, cardiopulmonary involvement, retroperitoneal involvement, as well as some other organs [14]. Although the disease can occur at any age, it has a higher frequency of occurrence between the age of 40 and 60. In addition to that, the severity of the disease can vary widely from patient to patient for instance, some patients may complain of few or no complications while others on the other hand might present with very serious problems that can be life-threatening [13].

\section{The clinical presentation of a patient with ECD depends on the site of involvement}

Bone: Patients with ECD most commonly show signs of bone involvement especially in the form of juxta-articular pain in the lower extremities. The infiltration of the histiocytes leads to the development of osteosclerosis and an increase in the bone density [15]. Furthermore, it is very common that patients would present with bilateral and symmetric osteosclerosis of the diaphysis and $83 \%$ of cases might also have the metaphysis affected. ECD most commonly affects the long bones of the arms and legs; however, there have also been few cases that presented with jaw pain [16]. A number of cases have been asymptomatic in which the bone involvement was detected during radiography for some other condition.

Heart: ECD can present with cardiovascular involvement in the form of either periaortic fibrosis, periarterial infiltration of the coronaries, or pericardial effusion. Additionally, patients can also suffer from various types of valve abnormalities and conduction defects. A study involving 37 patients who were screened with cardiac MRI and ECG showed that $70 \%$ of those patients had abnormal heart images and about $32 \%$ of them had abnormal ECGs. According to this study, half of the patients presented with a pseudotumor in the right heart [13]. Cardiac involvement in ECD tends to be life-threatening and is seen to be the cause of death in those patients.

Lungs: About $46 \%$ of ECD cases present with pulmonary involvement [17]. It is the progressive pulmonary fibrosis that can cause the patient to complain of dyspnea with exertion, a dried cough, or an impaired oxygen saturation of the blood [13]. High resolution chest tomography (HRCT) reveals signs of pleural effusion, lung cysts, centrilobular nodular opacities, or ground glass opacities. In addition to that, bronchial lavage would indicate the presence of macrophages and foamy histiocytes in the fluid [17]. All in all, knowing that ECD can present as interstitial lung disease especially in the form of either interstitial pneumonitis or pulmonary Langerhans cell histiocytosis allows good evaluation and judgment of the best ways to approach ECD therapeutically.

Nervous system: Neurological complications are identified in about $41 \%$ of ECD cases [18]. One of the most common manifestations is periorbital which is presented in the form of exophthalmoses that may or may not be associated with vision loss. This might also be associated with xanthelasma detected on the eyelids [19]. Some other common locations include the pituitary, brain parenchyma, meninges, and paranasal sinus. In 
the case where the pituitary gland is affected, patients present with diabetes insipidus experiencing excessive thirst and urination [20] Furthermore, since ECD can infiltrate the dura mater of the brain, it can be confused with meningitis. ECD can sometimes be associated with cognitive impairment presented as diffuse, bihemispheric reduction in cortical thickness [21].

Other organs: ECD can present with other organ involvement. For instance, the retro peritoneum can be affected leading to the formation of lesions that surround the kidneys consequently leading to renal impairment [22]. Skin involvement in ECD is not very common, however it would usually present as xanthelasma which mostly occurs on the eyelids. Other organs which might be affected in ECD include the breast, liver, spleen, and thyroid.

Diagnosis: Not only is it difficult to diagnose ECD because different patients may have different symptoms and signs, but also because patients may have other syndromes overlapping. For instance, a study done has revealed that more than a tenth of the patients with ECD were reported to also have Langerhans Cell Histiocytosis $[2,10]$. However, diagnosis could still be achieved. The best method to start diagnosis is usually through biopsies. Biopsies could give histologic, genetic, and immunophenotypic information. ECD histiocytes cause tissues to be foamy, lipidladen, and fibrotic. Histologically, they stain positively for factor XIIIa, CD68, CD163. They do not stain (negative) to CD1a, langerin, and S100 (only in rare cases) - giving a differentiation factor from Langerhans Cell Histiocytes.

Clinical manifestations of ECD are usually bilateral, which distinguishes it from POEMS syndrome and Paget's disease. Furthermore, ECD has low to none existent hemophagocytic activity in the biopsy due to, mainly, fibrosis. This factor separates ECD cases from hemophagocytic lymphohistiocytosis. ECD can also be misdiagnosed for diseases such as juvenile xanthogranuloma (JXB) and Rosai-Dorfman disease- both of which could be told apart, depending on the disease, either through severity and characteristics of symptoms, or histologically $[1,2,8,10]$.

Treatment: To this day, there is not a definitive standardized treatment regimen for patients with ECD. The use of interferon alpha tends to be the first line treatment option for patients who have symptomatic ECD with wild type BARF. The patient would receive a pegylated interferon at a dose of 135 micrograms per week which is then titrated upwards. If the patient does not respond to the pegylated interferon, then conventional interferon is started. Contrary to that, patients who show contraindication to interferon alpha are started on a BRAF inhibitor (Vemurafenib). Vemurafenib is a potent inhibitor of the kinase in the mutant BRAF. This drug is commonly used to treat metastatic melanomas, and recently studies have shown its effectiveness in the treatment of ECD in patients with intolerance to interferon or mutation in the BRAF V600E [23]. In the case of intolerance to Vemurafenib, a MEK inhibitor (Tramitinib) can be used. In some cases glucocorticoids are used. However, they have not shown to have any proven survival benefits. Glucocorticoids are used for patients presenting with mild symptoms or are not able to tolerate any of the previously mentioned aggressive treatments [24].

Management can be organ specific to relieve symptoms, like dieresis, fluid drainage from serious cavities, pleurocentesis for pleural effusion, pericardiocentesis for pericardial effusion and tamponade. Since some ECD cases can be asymptomatic, it is suggested to follow up with the patient for a specific period of time. The follow up regimen basically includes performing examinations to check the viability of organ and progression of disease. Affected organs should be screened every 3 to 6 months until stability is recorded. The prognosis for the disease is still poor due to the unavailability of a cure for ECD. Treatments such as interferon alpha have shown to increase survival rate by 5 year.

Plus the introduction of BRAF inhibitors has shown improvement in the prognosis of various cases where there is mutation in the BRAF gene. There are researches going on regarding the use of certain drugs for the treatment of ECD such as infliximab and anakinra which cause inhibition of cytokines as well as serine/ threokinase inhibitor.

\section{Conclusion}

Erdheim Chester Disease is a very rare condition characterized by histiocytosis and associated with various organ involvements. Recognizing the intricacy and the various complexities of this disease allow us to better recognize and assess the condition and consequently being able to proceed with the best treatment action. Management can be organ specific to relieve symptoms, like in our case pericardiocentesis for pericardial effusion and tamponade. Ongoing and future researches might expand our understanding of this intriguing syndrome.

\section{References}

1. What is Erdheim-Chester Disease?

2. http://www.mayoclinicproceedings.org/article/S0025-6196 (14)00144-X/fulltext\#sec1

3. https://www-uptodate-com.ezproxy.rcsi-mub.com/contents/ erdheim-chester-disease?

4. Wilejto M, Abla O (2012) Langerhans cell histiocytosis and ErdheimChester disease. Curr Opin Rheumatol 24(1): 90-96.

5. https://www.histio.org/page

6. https://rarediseases.info.nih.gov/diseases/6369/erdheim-chesterdisease

7. https://ghr.nlm.nih.gov/gene/BRAF

8. https://www-uptodate-com.ezproxy.rcsi-mub.com/contents/ erdheim-chester-disease? http://www.orpha.net/consor/cgi-bin/ Disease_$=$ Erdheim-Chester

9. http://www.orpha.net/consor/cgi-bin/

10. Eli L Diamond, Lorenzo Dagna, David M Hyman, Giulio Cavalli, Filip Janku, et al. (2014) Consensus Guidelines for the Diagnosis and Clinical Management of Erdheim-Chester Disease, Blood. 
11. Munoz J, Janku F, Cohen PR, Kurzrock R (2014) Erdheim-Chester Disease: Characteristics and Management. Mayo Clin Proc 89(7): 985996.

12. BRAF (2017) B-Raf proto-oncogene, serine/threonine kinase.

13. Sedrak P, Ketonen L, Hou P, Guha Thakurta N, Williams MD, et al (2011) Erdheim-Chester Disease of the Central Nervous System: New Manifestations of a Rare Disease. AJNR Am J Neuroradiol 32(11): 2126-2131.

14. https://ghr.nlm.nih.gov/condition/erdheim-chester-disease

15. https://www-uptodate-com.proxy.library.rcsi.ie/contents/ image?imageKey=HEME chester

16. https://www-uptodate-com.proxy.library.rcsi.ie/contents/erdheimchester-disease/abstract/1

17. Arnaud L, Gorochov G, Charlotte F, Lvovschi V, Parizot C, et al. (2011) Systemic perturbation of cytokine and chemokine networks in Erdheim-Chester disease: a single-center series of 37 patients. Blood 117(10): 2783-2790.

18. Brodkin CL, Wszolek ZK (2006) Neurologic presentation of ErdheimChester disease. Neurol Neurochir Pol 40(5): 397-403.

19. Cavalli G, Guglielmi B, Berti A, Campochiaro C, Sabbadini MG, et al. (2013) The multifaceted clinical presentations and manifestations of Erdheim-Chester disease: comprehensive review of the literature and of 10 new cases. Ann Rheum Dis 72(10): 1691-1695.

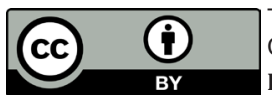

This work is licensed under Creative Commons Attribution 4.0 License

DOI:-10.19080/JOCCT.2017.07.555709
20. Diamond EL, Hatzoglou V, Patel S, Abdel Wahab O, Rampal R, et al. (2016) Diffuse reduction of cerebral grey matter volumes in ErdheimChester disease. Orphanet J Rare Dis 11(1): 109.

21. Surabhi VR, Menias C, Prasad SR, Patel AH, Nagar A, et al. (2008) Neoplastic and non-neoplastic proliferative disorders of the perirenal space: cross-sectional imaging findings. Radiographics Aug 28(4): 1005-1017.

22. Barnes PJ, Foyle A, Haché KA, Langley RG, Burrell S, et al. (2005) Erdheim-Chester disease of the breast: a case report and review of the literature. Breast J 11(6): 462-467.

23. Haroche J, Cohen Aubart F, Emile JF, Arnaud L, Maksud P, et al. (2013) Dramatic efficacy of vemurafenib in both multisystem and refractory Erdheim-Chester disease and Langerhans cell histiocytosis harboring the BRAF V600E mutation. Blood 121(9): 1495-1500.

24. Arnaud L, Hervier B, Néel A, Hamidou MA, Kahn JE, et al. (2011) CNS involvement and treatment with interferon- $\alpha$ are independent prognostic factors in Erdheim-Chester disease: a multicenter survival analysis of 53 patients. Blood 117(10): 2778-2782.

\section{Your next submission with Juniper Publishers} will reach you the below assets

- Quality Editorial service

- Swift Peer Review

- Reprints availability

- E-prints Service

- Manuscript Podcast for convenient understanding

- Global attainment for your research

- Manuscript accessibility in different formats ( Pdf, E-pub, Full Text, Audio)

- Unceasing customer service

Track the below URL for one-step submission https://juniperpublishers.com/online-submission.php 